\title{
THE DETERMINATION OF ALL SHEFFER FUNCTIONS IN 3-VALUED LOGIC, USING A LOGICAL COMPUTER
}

\section{ERIC FOXLEY}

This paper describes the method by which the results of a paper by Martin ${ }^{1}$ were used to enable the set of all Sheffer functions in 3-valued logic to be determined by the Nottingham University Logical Computer, ${ }^{2}$ and how the computer demonstrated that Martin's condition of co-substitution is superfluous.

We will use the symbolism of Jan Kukasiewicz, and represent the functors of non-implication, joint denial, non-equivalence and incompatibility by the letters $B, J, R, S$ respectively. The conditioned disjunction $[p, q, r]$ will be written $D q p r$, the change in the order of the variables being made as a matter of convenience. We will abbreviate any functor $F$ written consecutively $i$ times by $F^{i}$.

To solve problems in 3-valued logic on a 2-state computer, we represent each 3-valued variable by two 2-valued variables. The truth-values $1,2,3$ of a variable $p$ are represented by the assignments $T, T ; T, F ; F, F$ respectively to the two 2-valued variables $p_{1}$ and $p_{2}$. The pair FT is taken as meaningless. (If we were to follow exactly a method already suggested by Rose, ${ }^{3}$ we would represent the truth-value 3 by both of the pairs $F T$ and $F F$. If this method is adopted, any solution to the problem in which the truth-value 3 appears $i$ times will be found in $2^{i}$ different 2 -valued forms. To avoid recording this solution $2^{i}$ times, one of the pairs $F T, F F$ must be chosen to represent the truth-value 3 in solutions, and the recording mechanism must be inhibited whenever any of the variables to be recorded is represented by the other pair. There is now no point in defining the latter pair to represent any particular 3-valued truth-value, since the results obtained when this pair occurs are not recorded. The pair $F F$ was chosen to represent the truth-value 3 in solutions, since the pairs TT, TF, FF are more easily distinguished from each other in written solutions than the pairs $T T, T F, F T$.)

For any 3-valued two-variable functor $F p q$ we will define $r, s, t, \ldots, z$ to be propositions taking the truth-values of $F 11, F 12, F 13, F 21, \ldots, F 33$, where $1,2,3$ are logical constants taking the truth-values $1,2,3$ respectively. We will consider each of Martin's conditions in turn. 


\section{PROPER CLOSING}

The following table shows the conditions required for each of the possible subsets of truth-values not to be closed under Fpq.

\begin{tabular}{|c|c|l|}
\hline Subset & Condition for non-closure & Representation in computer \\
\hline 1 & $r \neq T 1$ & $N r_{2}$ \\
2 & $z \neq T 2$ & $E v_{1} v_{2}$ \\
3 & $z \neq T_{T}$ & $z_{1}$ \\
3,2 & At least 1 of $v, w, y, z=T_{T} 1$ & $A^{3} v_{2} w_{2} y_{2} z_{2}$ \\
1,3 & At least 1 of $r, t, x, z=T_{T}$ & $A{ }^{3} B r_{1} r_{2} B t_{1} t_{2} B x_{1} x_{2} B z_{1} z_{2}$ \\
2,1 & At least 1 of $r, s, u, v=T_{T}^{3}$ & $N K^{3} r_{1} s_{1} u_{1} v_{1}$ \\
\hline
\end{tabular}

We will number these conditions $1, \ldots, 6$ respectively. They must all be satisfied for $F p q$ to be a Sheffer function.

\section{$t$-CLOSING}

There are only two $t$-functions in 3-valued logic. Their truth-tables are

\begin{tabular}{c|c|c}
$p$ & $T p$ & $U p$ \\
\hline 1 & 2 & 3 \\
2 & 3 & 1 \\
3 & 1 & 2
\end{tabular}

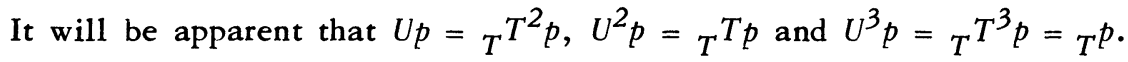
For all values of $i$ and $j$, functions of the type $T^{i} p, U^{j} p$ can therefore be obtained in the form $T^{k} p$, where $k=0,1$ or 2 , so we need not consider the function $U$, or powers of $T$ higher than the second.

\section{THEOREM}

$F p q$ is $t$-closing if and only if there exist $i, j, k$ such that $F p p=T^{T} T^{i}$, $F p T p=T_{T} T^{j} p, F T p p^{\prime}=T_{T} T^{k}$, where $i, j, k \in\{0,1,2\}$

Proof of necessity: Trivial, from definition of $t$-closure.

Proof of sufficiency: The following functions also take the truth-table of some power of $T$ :

$$
\begin{aligned}
& F T p T p={ }_{T} T^{i} T p={ }_{T} T^{i+1} T p \\
& F T^{2} p T^{2} p={ }_{T} T^{i} T^{2} p={ }_{T} T^{i+2} p \\
& F T p T^{2} p={ }_{T} T^{j} T p={ }_{T} T^{j+1} p \\
& F T^{2} p p={ }_{T} F T^{2} p T T^{2} p={ }_{T} T^{j} T^{2} p={ }_{T} T^{j+2} p \\
& F T^{2} p T p={ }_{T} T^{k} T p={ }_{T} T^{k+1} p \\
& F p T^{2} p={ }_{T} F T T^{2} p T^{2} p={ }_{T} T^{k} T^{2} p={ }_{T} T^{k+2} p
\end{aligned}
$$


Hence, for all $l, m$ there is an $n$ such that $F T^{l} p T^{m} p={ }_{T} T^{n} p$, and $F p q$ is $t$ closing.

The truth-values of $F p p, F p T p, F T p p$ for $p=T_{T}^{1,2,3}$ are determined by the propositions shown below:

\begin{tabular}{c|c|c|c}
$p$ & $F p p$ & $F p T p$ & $F T p p$ \\
\hline 1 & $r$ & $s$ & $u$ \\
2 & $v$ & $w$ & $y$ \\
3 & $z$ & $x$ & $t$
\end{tabular}

Hence for Fpq to be $t$-closing, the triples $r, v, x ; s, w, x ; u, y, t$ must each take one of the assignments of truth-values $1,2,3 ; 2,3,1 ; 3,1,2$.

The condition that the triple $s, w, x$ takes one of these assignments can be expressed: (If $s=T_{T} 1$, then $w=T^{2}$ and $x=T_{T}$ ) or (if $s=T_{T}^{2}$, then $w=T_{T}^{3}$ and $x=T_{T}$ ) or (if $s=T^{3}$, then $w=T_{T}^{1}$ and $x=T_{T}^{2}$ ). The latter parts of the se three disjuncts can be expressed $B \dot{B} w_{1} w_{2} x_{1}, B x_{2} w_{1}, K B x_{1} x_{2} w_{2}$ respectively, and are required to take the truth-value $T$ when $s_{1} s_{2}$ take the assignments $T T, T F, F F$ respectively. The condition can therefore be expressed

$$
D s_{1} D s_{2} B B w_{1} w_{2} x_{1} B x_{2} w_{1} K B x_{1} x_{2} w_{2} \text {. }
$$

For Fpq to be a Sheffer function, it must not be $t$-closing, i.e. at least one of the triples $s, w, x ; t, u, y ; r, v, z$ must take some assignment other than $1,2,3 ; 2,3,1 ; 3,1,2$. This can be expressed as the disjunction of the three conditions

$$
\begin{aligned}
& D s_{1} D s_{2} C B w_{1} w_{2} x_{1} C x_{2} w_{1} S B x_{1} x_{2} w_{2} \\
& D u_{1} D u_{2} C B y_{1} y_{2} t_{1} C t_{2} y_{1} S B t_{1} t_{2} y_{2} \\
& D r_{1} D r_{2} C B v_{1} v_{2} z_{1} C z_{2} v_{1} S B z_{1} z_{2} v_{2} .
\end{aligned}
$$

We will number these conditions $7 a, 7 b, 7 c$ respectively, and their disjunction as number 7 .

\section{PROPER SUBSTITUTION}

There are three possible decompositions of the three truth-values into less than three classes. They are $\{1\},\{2,3\} ;\{2\},\{3,1\}$ and $\{3\},\{1,2\}$. The condition for proper substitution for the first of these decompositions is that all or none of each of the sets of variables $v, w, y, z ; s, t ; u, x$ should take the truth-value 1 . The conditions for the three separate sets can be expressed $C A^{3} v_{2} w_{2} y_{2} z_{2} K^{3} v_{2} w_{2} y_{2} z_{2}, E s_{2} t_{2}, E u_{2} x_{2}$ respectively. For Fpq to be a Sheffer function, we require the failure of at least one of these conditions, which can be expressed

$$
A^{2} B A^{3} v_{2} w_{2} y_{2} z_{2} K^{3} v_{2} w_{2} y_{2} z_{2} R s_{2} t_{2} R u_{2} x_{2} \text {. }
$$

For the second decomposition the sets of variables are $r, t, x, z ; s, y$; $u, w$ and the condition becomes 


$$
\begin{gathered}
A^{2} B A^{3} B r_{1} r_{2} B t_{1} t_{2} B x_{1} x_{2} B z_{1} z_{2} K^{3} B r_{1} r_{2} B t_{1} t_{2} B x_{1} x_{2} B z_{1} z_{2} R B s_{1} s_{2} \\
B y_{1} y_{2} R B u_{1} u_{2} B w_{1} w_{2} .
\end{gathered}
$$

For the third decomposition, the sets of variables are $r, s, u, v ; t, w$; $x, y$ and the condition is

$$
A^{2} B A^{3} r_{1} s_{1} u_{1} v_{1} K^{3} r_{1} s_{1} u_{1} v_{1} R t_{1} w_{1} R x_{1} y_{1}
$$

These three conditions, which we will number $8,9,10$, must all be satisfied for Fpq to be a Sheffer function.

\section{CO-SUBSTITUTION}

If $F p q$ is to satisfy the co-substitution property for the decomposition $\{1\},\{2,3\}$ one of the sets $u, x ; s, t$ must all take the truth-value 1 , and the other set must all take the truth-values 2 or 3, with similar restrictions on the sets $v, w, y, z ; r$. Under these conditions, $F p q$ will also satisfy the proper substitution property. Hence, since we have already ensured that $F p q$ does not satisfy the proper substitution property, it cannot satisfy the co-substitution property for the above decomposition, or for any other decomposition in to two classes.

For decomposition of the truth-values into three classes, we can replace the " $\sim$ " sign by " $=T$ ", since each class contains only one member.

We will show that, in this case, the co-substitution property requires either each row of the truth-table to contain three different entries and each column three identical entries, or vice versa.

Let the triples $a, b, c ; d, e, f ; g, b$, $i$ each be rearrangements of the logical constants $1,2,3$.

The entries in the truth-table cannot all be the same, since $F 11={ }_{T} F 22$ would violate the co-substitution requirements. Hence there must exist a row or column containing two different entries. We will assume that there is a row containing two different entries, and will show that it must contain three different entries, and that each column must contain three identical entries. The case starting with a column containing two different entries can be argued similarly.

From our assumption above, we can choose $a, d, e, g, b$ such that

$$
F a d=T^{g} \text { and } F a e=T_{T}^{b} .
$$

Since $\mathrm{Fad}={ }_{T} g$, the co-substitution property requires that

$$
\text { Fbe, Fbf, Fce, Fcf } \neq{ }_{T} g \ldots \ldots \ldots \ldots \ldots \ldots
$$

and similarly, since Fae $={ }_{T}$,

$$
F b d, F b f, F c d, F c f \neq{ }_{T} b \ldots \ldots \ldots \ldots \ldots \ldots \ldots \ldots \ldots
$$

From 1 and 2,

$$
F b f={ }_{T} F c f=T^{i} .
$$


Since $F b f={ }_{T} i$, we must have

Fad, Fae, Fcd, Fce $\neq{ }_{T}{ }^{i} \ldots \ldots \ldots \ldots \ldots \ldots$

Fad and Fae satisfy this condition, and from 2 and 3

$$
F c d=T^{g}
$$

and from 1 and 3

$$
F c e=T^{b}
$$

Since $F c f=T^{i}$, we must have

Fad, Fae, Fbd, Fbe $\neq{ }_{T}{ }^{i} \ldots \ldots \ldots \ldots \ldots$

Fad and Fae satisfy this condition, and from 2 and 4

$$
F b d={ }_{T} g
$$

and from 1 and 4

$$
F b e=T^{b}
$$

Since $F b d={ }_{T} g$, we must have

$$
\text { Fae, Faf, Fce, Fcf } \neq{ }_{T} g \ldots \ldots \ldots \ldots \ldots
$$

Fae, Fce and Fcf satisfy this condition.

Since $F b e=T_{T}$, we must have

$$
F a d, F a f, F c d, F c f \neq{ }_{T} b \ldots \ldots \ldots \ldots
$$

$F a d, F c d, F c f$ satisfy this condition.

From 5 and 6,

$$
F a f=T^{i}
$$

Hence

$$
\begin{aligned}
& F a d={ }_{T} F b d={ }_{T} F c d={ }_{T} g \\
& F a e={ }_{T} F b e={ }_{T} F c e={ }_{T} b \\
& F a f={ }_{T} F b f={ }_{T} F c f={ }_{T}{ }^{i}
\end{aligned}
$$

giving the required result.

The co-substitution requirement can now be expressed as follows: $r, v, z$ must take different truth-values, and either each row, or each column, must contain three identical entries. The first part of this condition can be expressed "If $r={ }_{T} 1, v, z$ take the assignment 2,3 or 3, 2; if $r={ }_{T}{ }^{2}, v, z$ take the assignment 3, 1 or 1,3 ; if $r=T^{3}, v, z$ take the assignment 1,2 or $2,1$. " This is expressed in the computer as 


$$
D r_{1} D r_{2} K J v_{2} z_{2} R v_{1} z_{1} A B v_{2} z_{1} B z_{2} v_{1} K^{2} v_{1} z_{1} R v_{2} z_{2}
$$

The second part of the condition is expressed

$$
\begin{gathered}
A K^{11} E r_{1} s_{1} E r_{2} r_{2} E r_{1} t_{1} E r_{2} t_{2} E v_{1} u_{1} E v_{2} u_{2} E v_{1} w_{1} E v_{2} w_{2} E x_{1} z_{1} E x_{2} z_{2} \\
E y_{1} z_{1} E y_{2} z_{2} K^{11} E r_{1} u_{1} E r_{2} u_{2} E r_{1} x_{1} E r_{2} x_{2} E v_{1} s_{1} E v_{2} s_{2} E v_{1} y_{1} E v_{2} y_{2} \\
E z_{1} t_{1} E z_{2} t_{2} E z_{1} w_{1} E z_{2} w_{2} .
\end{gathered}
$$

For Fpq to be a Sheffer function, at least one of the above two conditions must fail. We will number this condition (the above two parts connected by the functor $S$ ) 11 .

We now have 11 conditions to feed into a conjunction mechanism leading to the final output. To these we must add the nine conditions (numbered 12 to 20) $C r_{2} r_{1}, C s_{2} s_{1}, \ldots, C z_{2} z_{1}$ to ensure that no many-valued variable is represented in a solution by the pair FT. To complete the problem, all $2^{18}$ possible assignments of truth-values to $r_{1}, r_{2}, s_{1}, \ldots, z_{2}$ are tested in succession, and all those for which the final output takes the truth-value $T$ are recorded.

The amount of equipment and length of time necessary to complete the problem can be reduced considerably by using the following simplifications.

All solutions in which $r=T^{3}$ can be obtained from ones in which $r=T^{2}$ by interchanging the truth-values 2 and 3 everywhere. Since we are not interested in truth-tables in which $r=T^{1}$, (the proper closing property requires $r \neq T^{1)}$, we can fix the truth-value of $r$ at 2, and will obtain exactly half the solutions. If we introduce the two-valued logical constants 0,1 taking the truth-values $F, T$ respectively, we can replace $r_{1}, r_{2}$ by 1,0 respectively in all the above conditions.

As we are not interested in solutions in which $v=T^{2}$, we can replace $v_{2}$ by $v_{1}$ everywhere, and thus ensure that $v$ can only take the truth-values 1 and 3.

Similarly, since we are only interested in truth-tables in which $z=T^{1}$ or 2, we can fix the truth-value of $z$ at $T$, and avoid scanning the unnecessary assignments $F T, F F$ to $z_{1} z_{2}$.

We need now scan only $2^{14}$ assignments instead of $2^{18}$, since four variables have been omitted from the set over which we scan. We will now examine the effect of the substitutions $r_{1} / 1, r_{2} / 0, v_{2} / v_{1}, z_{1} / 1$ on the above 19 conditions.

Condition 1: $N r_{2}=T^{1}$, therefore this condition can be omitted.

Condition 2: $E v_{1} v_{1}=T^{1}$, and this condition can be omitted.

Condition 3: $z_{13}=T^{1}$, and this condition can be omitted.

Condition 4: $A^{3} v_{1} w_{2} y_{2} z_{2}$

Condition 5: $A^{3} B 10 \ldots=T_{T}$, and this condition can be omitted.

Condition 6: $N K^{3} 1 s_{1} u_{1} v_{1}={ }_{T} N K^{2} s_{1} u_{1} v_{1}$

Conditions $7 \mathrm{a}, 7 \mathrm{~b}$ are unchanged.

Condition 7c: $D 1 D 0 C B v_{1} v_{1} 1 C z_{2} v_{1} S B 1 z_{2} v_{1}={ }_{T} C z_{2} v_{1}$ 


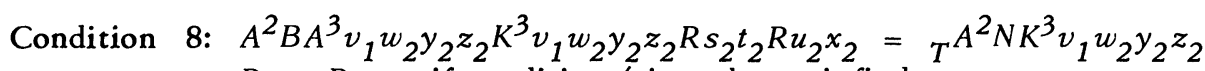
$R s_{2} t_{2} R u_{2} x_{2}$ if condition 4 is to be satisfied.

Condition 9: $A^{2} B 1 K^{3} 1 B t_{1} t_{2} B x_{1} x_{2} B 1 z_{2} R B s_{1} s_{2} B y_{1} y_{2} R B u_{1} u_{2} B w_{1} w_{2}=T^{A^{2}}$ $N K^{2} B t_{1} t_{2} B x_{1} x_{2} N z_{2} R B s_{1} s_{2} B y_{1} y_{2} R B u_{1} u_{2} B w_{1} w_{2}=T^{A} C K B t_{1} t_{2}$ $B x_{1} x_{2} z_{2} R B s_{1} s_{2} B y_{1} y_{2} R B u_{1} u_{2} B w_{1} w_{2}$

Condition 10: $A^{2} B 1 K^{2} s_{1} u_{1} v_{1} \ldots=T^{1}$ if condition 6 is satisfied, and this condition can be omitted.

Condition 11: The first part becomes $A B v_{1} 1 B z_{2} v_{1}={ }_{T} B z_{2} v_{1}$. The second part becomes $A K^{6} B s_{1} s_{2} B t_{1} t_{2} C A^{4} u_{1} u_{2} v_{1} w_{1} w_{2} K^{4} u_{1} u_{2} v_{1} w_{1} w_{2}$ $E x_{2} z_{2} E y_{2} z_{2} x_{1} z_{1} K^{6} B u_{1} u_{2} B x_{1} x_{2} C A^{4} s_{1} s_{2} v_{1} y_{1} y_{2} K^{4} s_{1} s_{2} v_{1} y_{1} y_{2}$ $E t_{2} z_{2} E w_{2} z_{2} t_{1} w_{1}$.

Conditions 12, 16, 20 now all take the truth-value $T$, and can be omitted.

Conditions 13,14,15,17, 18, 19 are unchanged.

We are now left with 12 conditions to feed into the conjunction mechanism leading to the final output. The time taken to test the $2^{14}$ assignments is about $1 \frac{1}{2}$ hours.

It was decided to use the computer to test whether the conditions 4,6 , $7,8,9,11$ were independent of each other. Let us represent these conditions by the variables $P_{1}, \ldots, P_{6}$, and the conjunction of conditions 13 , $14,15,17,18,19$ by the variable $Q$. To test the independence of condition $P_{i}$, we note that if the other five conditions and $Q$ all take the truth-value $T$ at some time when $P_{i}$ takes the truth-value $F$, omission of $P_{i}$ would cause the output to take the truth-value $T$, giving a false indication of a Sheffer function. The condition $P_{i}$ must then be independent of the other conditions, since it cannot be omitted. This can be expressed

$$
K B\left(\prod_{\substack{1 \leq j \leq 6 \\ j \neq i}} P_{j}\right) P_{i} Q .
$$

(The variable $Q$ must be included to ensure a meaningful assignment to $s_{1}, \ldots, z_{2}$.)

These six conditions $(i=1, \ldots, 6)$ are each fed to the trigger input of a flip-flop, ${ }^{4}$ so that the triggering of the $i$-th flip-flop indicates the independence of condition $P_{i}$.

Since it was desirable that the computer record truth-tables indicating the independence of each condition, the logical arrangement of figure 1 was set up for each condition $P_{i}$. There are six such arrangements, and the outputs from the six upper B-mechanisms are taken to a six input disjunction mechanism. The lower flip-flop is the one mentioned earlier, which indicates the independence of condition $P_{i}$. The output from the lower B-mechanism triggers the upper flip-flop at the end of the first pulse to the trigger input of the lower flip-flop. The output of the upper B-mechanism thus takes the truth-value $T$ only during this first pulse, but not during any later pulses to the lower flip-flop's trigger input. If the machine is now made to record the truth-values of $s_{1}, \ldots, z_{2}$ whenever the disjunction of these six outputs 
Figure 1

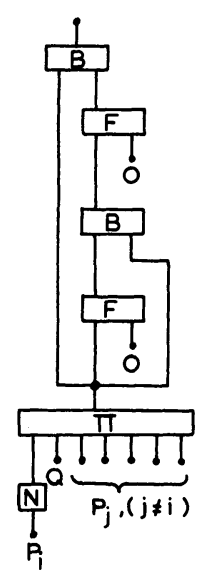

takes the truth-value $T$, it will record the first truth-table demonstrating the independence of each condition which is independent.

After scanning all assignments to $s_{1}, \ldots, z_{2}$, the lower flip-flop corresponding to $P_{6}$ had not been triggered, but truth-tables had been recorded demonstrating the independence of each of the other conditions. These are

Figure 2

\begin{tabular}{|c|c|c|}
\hline Truth-table & $\begin{array}{c}\text { Satisfies all of } \\
P_{1}, \ldots, P_{6} \text { except }\end{array}$ & $\begin{array}{l}\text { Satisfies none of Martin's } \\
\text { conditions except }\end{array}$ \\
\hline $\begin{array}{lll}2 & 3 & 3 \\
3 & 3 & 3 \\
1 & 3 & 2\end{array}$ & $P_{1}$ & $\begin{array}{l}\text { Proper clo sing } \\
\text { Subset }\{2,3\}\end{array}$ \\
\hline $\begin{array}{lll}2 & 2 & 3 \\
2 & 1 & 3 \\
3 & 3 & 2\end{array}$ & $P_{2}$ & $\begin{array}{l}\text { Proper closing, } \\
\text { Subset }\{1,2\}\end{array}$ \\
\hline $\begin{array}{lll}2 & 3 & 3 \\
1 & 3 & 1 \\
2 & 2 & 1\end{array}$ & $P_{3}$ & $t$-closing \\
\hline $\begin{array}{lll}2 & 3 & 3 \\
3 & 1 & 1 \\
3 & 1 & 1\end{array}$ & $P_{4}$ & $\begin{array}{l}\text { Proper substitution, } \\
\text { Decomposition }\{1\},\{2,3\}\end{array}$ \\
\hline $\begin{array}{lll}2 & 3 & 2 \\
3 & 3 & 3 \\
2 & 1 & 2\end{array}$ & $P_{5}$ & $\begin{array}{l}\text { Proper substitution, } \\
\text { Decomposition }\{2\},\{1,3\}\end{array}$ \\
\hline
\end{tabular}


shown in figure 2. Hence the condition that $F p q$ has not the co-substitution property is contained in the other conditions, and can be omitted.

The scan over all assignments to $s_{1}, \ldots, z_{2}$ was repeated, and a similar method used to see if the condition $P_{6}$ was contained in any one of the other five conditions. It was found to be contained in condition $P_{3}$, the condition that $F p q$ should not be $t$-closing. A formal proof of this inclusion is given at the end of this paper.

The problem can now be completed using 36 universal decision elements, one 4-input disjunction mechanism (for condition 4) and one 11-input conjunction mechanism for the final output. The universal decision elements in the Nottingham University Logical Computer can be used as 3-input conjunction and disjunction mechanisms, and the use of the terms $K^{2}, A^{2}$ in the above conditions infers that a single element is used. In condition 8 we replace $N K^{3} v_{1} w_{2} y_{2} z_{2}$ by $S K^{2} v_{1} w_{2} y_{2} z_{2}$, this function being generated by two universal elements.

The problem was first run through just counting the number of solutions, without recording them. The number found was 1887 , in agreement with Martin, but too numerous for it to be practicable to record them all. The problem was therefore repeated for symmetric functions only, and all solutions actually recorded.

The determination of all symmetric functions can be approached in two ways. If the computer is already set up to solve the previous problem, we add the three conditions $s={ }_{T} u\left(K E s_{1} u_{1} E s_{2} u_{2}\right), t=T_{T} x$ and $w={ }_{T} y$ and proceed as above. Alternatively, we can replace $u_{1}, u_{2}, x_{1}, x_{2}, y_{1}, y_{2}$ in the above conditions by $s_{1}, s_{2}, t_{1}, t_{2}, w_{1}, w_{2}$ respectively, and reprogramme the computer with the new conditions, which are as follows:

Condition 4: $A^{2} v_{1} w_{2} z_{2}$

Condition 6: $N K^{2} s_{1} s_{1} v_{1}=T_{T} S s_{1} v_{1}$

Condition 7: $A D s_{1} D s_{2} C B w_{1} w_{2} t_{1} C t_{2} w_{1} S B t_{1} t_{2} w_{2} C z_{2} v_{1}$, since conditions 7a and $7 \mathrm{~b}$ are now identical.

Condition 8: $A^{2} N K^{3} v_{1} w_{2} w_{2} z_{2} R s_{2} t_{2} R s_{2} t_{2}={ }_{T} C K^{2} v_{1} w_{2} z_{2} R s_{2} t_{2}$

Condition 9: $A^{2} C K B t_{1} t_{2} B t_{1} t_{2} z_{2} R B s_{1} s_{2} B w_{1} w_{2} R B s_{1} s_{2} B w_{1} w_{2}={ }_{T}^{A C B t_{1} t_{2} z_{2}}$ $R B s_{1} s_{2} B w_{1} w_{2}$.

Conditions 15, 18, 19 are identical with conditions $13,14,17$ respectively, and can be omitted.

The total number of operations is now $2^{8}$, the eight variables being $s_{1}$, $s_{2}, t_{1}, t_{2}, v_{1}, w_{1}, w_{2}, z_{2}$. The number of universal decision elements required is 21 , together with one 8 -input conjunction mechanism for the final output.

We will now give a formal proof of the inclusion property demonstrated by the computer.

\section{THEOREM}

If Fpq has none of the properties of proper closing, $t$-closing and proper substitution, then it cannot bave the co-substitution property. 
The case when the three truth-values are decomposed in to two classes has been dealt with earlier in the paragraph on co-substitution.

For decomposition of the truth-values into three classes, the co-substitution property requires $r, v, z$ to take different truth-values, and since $F p q$ is not proper closing, $r \neq T_{T} 1, v \neq T_{T}^{2}, z \notin T^{3}$. The only possible assignments to $r, v, z$ are therefore $2,3,1$ and $3,1,2$. There are then four possible assignments to $r, \ldots, z$, namely

$$
\begin{aligned}
& r=T^{s}=T^{t=} T^{2,} \quad u=T^{v=} T^{w=\cdot} T^{3,} \quad x=T_{T}^{y=} T^{z=} T^{1 ;} \\
& r=T^{s=} T^{t=} T^{3,} \quad u=T^{v=} T^{w=} T^{1,} \quad x=T^{y=} T^{z=} T^{2 ;} \\
& r=T^{u=} T^{x=} T^{2,} \quad s=T^{v=} T^{y=} T^{3,} \quad t=T^{w=} T^{z=} T^{1 ;} \\
& r=T^{u=} T^{x=} T^{3,} \quad s=T^{v=} T^{y=} T^{1,} \quad t=T^{w=} T^{z}=T^{2 .}
\end{aligned}
$$

In all these cases, the triples $s, w, x ; t, u, y ; r, v, z$ each take one of the assignments $1,2,3 ; 2,3,1 ; 3,1,2$, and $F p q$ is $t$-closing. This contradicts our original assumption, and the theorem is thus proved.

\section{NOTES}

1. N. M. Martin, The Sheffer functions of 3-valued logic, The Journal of Symbolic Logic, vol. 19 (1954), pp. 45-51.

2. For the concept of a logical computer see D. B. McCallum and J. B. Smith, Mechanised reasoning-logical computers and their design, Electronic Engineering, vol. 23 (1951), pp. 126-33.

3. Alan Rose, Many-valued logical machines, Proc. Camb. Phil. Soc., vol. 54 (1958), pp. 307-21.

4. Alan Rose, The use of universal decision elements as flip-flops, Zeit. für Math. Log., vol. 4 (1958), pp. 169-74.

The University of Nottingham

Nottingham, England 